\title{
FILOSOFÍA Y LITERATURA \\ LA EXPERIENCIA ESTÉTICA \\ EN ESTANISLAO ZULETA
}

CRISTHIAN PERDIGÓN LESMES

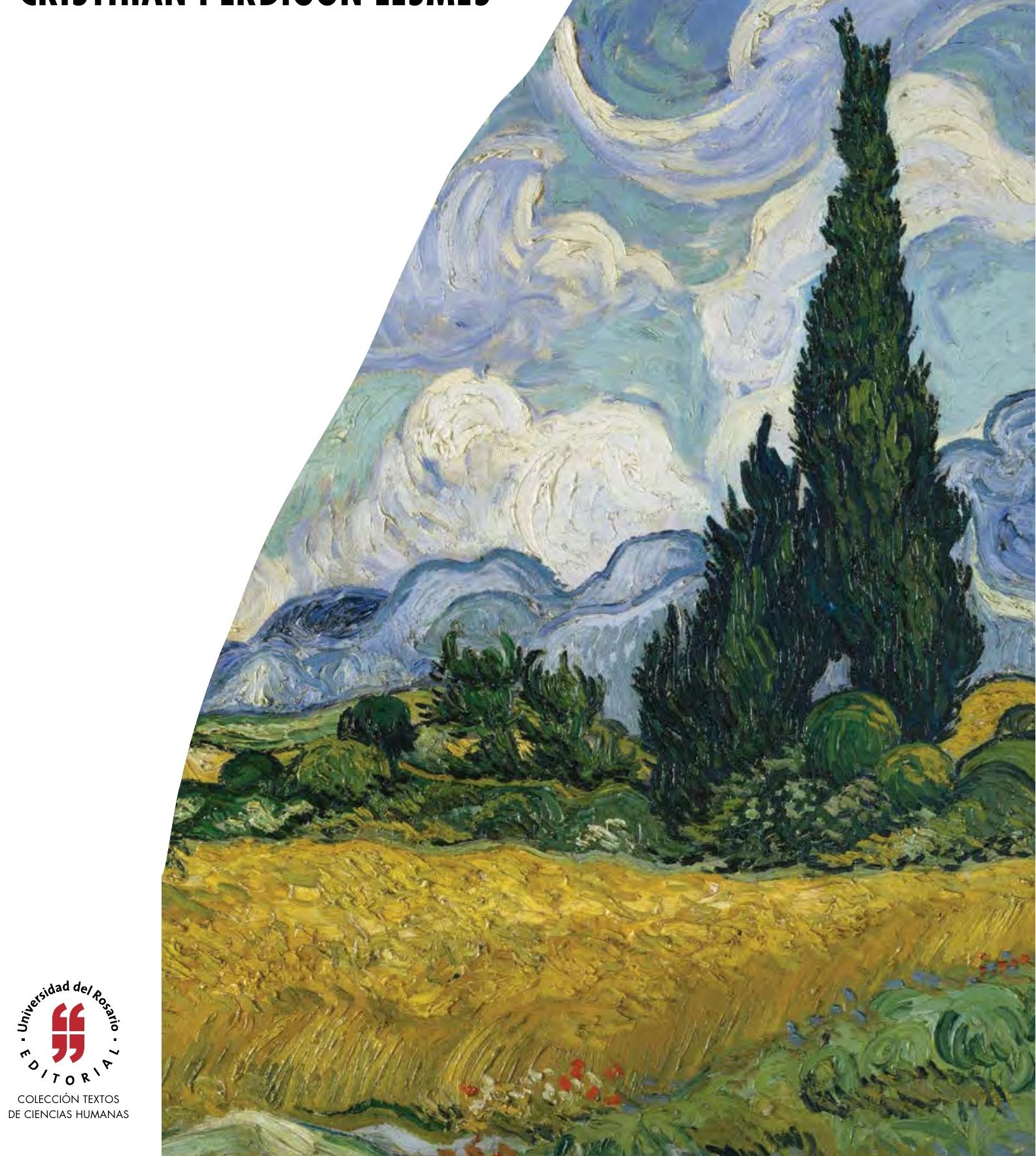




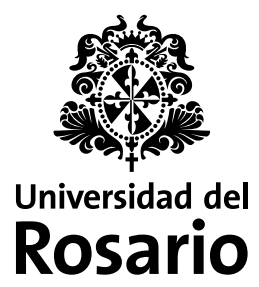





\section{Filosofía y literatura La experiencia estética en Estanislao Zuleta}




\section{Perdigón Lesmes, Cristhian}

Filosofia y literatura: la experiencia estética en Estanislao Zuleta / Cristhian Perdigón Lesmes. - Bogotá: Editorial Universidad del Rosario, Escuela de Ciencias Humanas, 2015.

viii, 98 páginas. - (Colección Textos de Ciencias Humanas)

Incluye referencias bibliográficas.

ISBN: 978-958-738-635-6 (rústica)

ISBN: 978-958-738-636-3 (digital)

Zuleta, Estanislao - 1935 - 1990 / Zuleta, Estanislao - 1935 - 1990 - Crítica e interpretación / Zuleta, Estanislao - 1935

- 1990 - pensamiento filosófico / Filosofía de la literatura / Crítica literaria / I. Título / II. Serie

801.95

SCDD 20

Catalogación en la fuente - Universidad del Rosario. Biblioteca

agh

Julio 7 de 2015

Hecho el depósito legal que marca el Decreto 460 de 1995 


\section{Filosofía y literatura La experiencia estética en Estanislao Zuleta}

Cristhian Perdigón Lesmes 


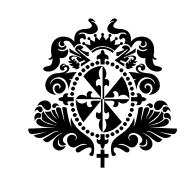

Colección Textos de Ciencias Humanas

(c) Editorial Universidad del Rosario

(c) Universidad del Rosario, Escuela de Ciencias Humanas

(c) Cristhian Perdigón Lesmes

Primera edición: Bogotá, D.C., agosto de 2015

ISBN: 978-958-738-635-6 (rústica)

ISBN: 978-958-738-636-3 (digital)

Coordinación editorial: Editorial Universidad del Rosario Corrección de estilo: Ella Suárez

Diagramación: Martha Echeverry

Montaje de cubierta: David Reyes

Impresión: Xpress. Estudio Gráfico y Digital S.A.

Editorial Universidad del Rosario

Carrera 7 No. 12B-41, of. 501 • Tel: 2970200 Ext. 7724

http://editorial.urosario.edu.co

Impreso y hecho en Colombia

Printed and made in Colombia

Fecha de evaluación: 29 de mayo de 2015

Fecha de aceptación: 19 de septiembre de 2015

Todos los derechos reservados. Esta obra no puede ser reproducida sin el permiso previo escrito de la Editorial Universidad del Rosario 


\section{Contenido}

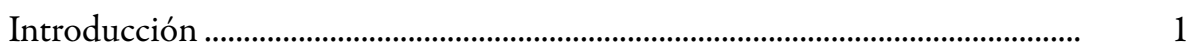

La "experiencia" personal.....................................................................................

Emancipación literaria ..............................................................................

Experiencia intencional, psicoanálisis y palabra ........................................ 20

La "ontología del presente": Modernidad y ethos crítico ....................................... 27

La novela como "aventura" .................................................................................. 39

"Aventura": don Quijote y su "salida" en busca del sentido ......................... 39

Transfiguración y sublimación: Kafka........................................................ 49

Thomas Mann y la ironía crítica ............................................................... $\quad 58$

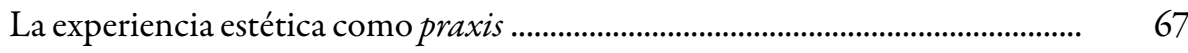

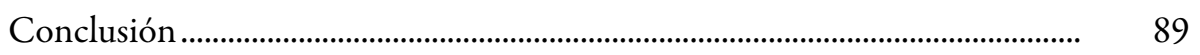

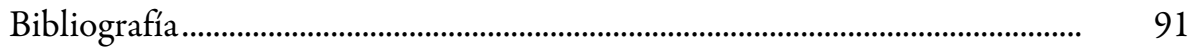

Publicaciones autógrafas de Estanislao Zuleta ........................................... 91

Publicaciones sobre Estanislao Zuleta......................................................... 92

Fuentes adicionales ................................................................................... 94 



\section{Introducción}

En cuanto manifestación discursiva y conceptual del pensamiento, la filosofía frecuentemente ha enfrentado la crítica de que en su pretensión por dar exhaustiva cuenta de lo que llamamos realidad se aleja de la más básica y espontánea experiencia humana al sobreponerle abstractos e ideales modelos de explicación. En consecuencia, tales modelos poco o nada dicen directamente de esas vivencias transhistóricas de hombres y mujeres a las que retrospectiva e históricamente denominamos cultura. Allende de dicha crítica, el arte, y en un muy destacado lugar la literatura, se ubica en una posición opuesta a los grandes sistemas filosóficos precisamente porque, como es el caso de cualquier novela, la realidad y la cultura se expresan como las vivencias de unos individuos enfrentados al complejo mundo real. Así presentadas, filosofía y literatura parecen oponerse formal y fundamentalmente. No obstante, de Platón a Borges la historia de la filosofía y la de la literatura no han dejado de cruzar sus sinuosos caminos y han dado lugar a una simbiótica relación que, a pesar de múltiples tensiones y desencuentros, está motivada por el interés común en la experiencia humana y sus diversas formas de configurar la realidad.

La forma en que Estanislao Zuleta supo articular filosofía y literatura, destacando sus respectivos alcances, cooperaciones y desacuerdos, es el tema que nos interesa abordar a continuación para resaltar de qué manera, en su particular y polémica forma de hacer filosofía, la literatura desempeñó un lugar tan fundamental que, si se deja de lado, la figura de Zuleta queda reducida a la de un intérprete o difusor de la filosofía continental. Si bien esto último es una valoración que con justicia se ha hecho del pensador colombiano, nos interesa ir mucho más allá para justificar la existencia de una filosofía zuletiana que, gracias a su indisoluble relación con la literatura y el arte, se inscribe en el proyecto contemporáneo que de Marx y Husserl a Foucault logró oponerse al idealismo abstracto.

Como se ha señalado en múltiples ocasiones, los más importantes aportes de Zuleta están relacionados con su juicioso estudio crítico y consecuente difusión 
de las obras de Marx y Freud. ${ }^{1}$ Para el presente estudio del pensamiento y la obra publicada de Zuleta, la articulación del materialismo marxista y el psicoanálisis freudiano también es central para la tesis que nos interesa defender, según la cual, producto de la relación que establece el pensador entre filosofía y literatura, es posible identificar una coherente teoría estética. La más terminada y sistematizada expresión de dicha teoría es una idea original de experiencia estética que conjuga los conceptos de elaboración y sublimación de Freud con la noción materialista de realidad de Marx, tal y como se manifiesta en los conceptos de historia y trabajo del autor de El capital. En términos más concretos, el concepto de experiencia estética que proponemos puede entenderse como la capacidad que ofrece la literatura y el arte en general de reconocer critica y autónomamente la realidad en el sentido de identidad cultural e individual.

Para reconstruir y hacer comprensible dicho concepto, nuestra exposición estará dividida en cuatro partes principales: una primera, donde a partir de la propia experiencia personal del autor con la literatura y la filosofía daremos cuenta de la forma en que se consolidó su propio pensamiento como una verdadera y autónoma emancipación que tuvo lugar gracias a su temprano encuentro con la literatura y el psicoanálisis. En este primer capítulo nos centraremos en la relación entre filosofía y literatura desde el concepto de Lewensbelt, de Husserl, para mostrar como modélica la experiencia personal de Zuleta respecto a la exigencia histórica de la filosofía contemporánea de inscribir la experiencia cotidiana del hombre en la justificación de la realidad. En procura de satisfacer este mismo punto, también veremos aquí cómo el psicoanálisis freudiano se emparenta con la literatura y la fenomenología, en cuanto reflexión y práctica lingüística, de forma tal que los aportes de Freud al pensamiento se ofrecen como una de las mejores propuestas filosóficas no abstractas de explicación de lo que constituye la realidad.

En el segundo capítulo, nuestro propósito será ubicar el pensamiento del colombiano en el contexto de la modernidad, para revelar cómo el vínculo que su filosofía establece con la literatura se inscribe en lo que Kant definió como crítica trascendental y cuya realización tuvo lugar en la crítica social que llevó a cabo la gran literatura universal de dicho periodo. En la segunda parte también veremos cómo las reflexiones del colombiano participan de la "ontología del presente" de Foucault, ya

1 Cf. Espinosa, “El psicoanálisis...” y “Encuentro...”; Giraldo, “E. Z.: un pensador...”; Navarro, “E. Z., el pensamiento...”; Salazar, “Entre Marx y...”; Valencia, “E. Z. y el...”. 
que, como el pensador francés, Zuleta parte de una lectura de Kant y de la filosofía crítica que le otorga un lugar central tanto a la literatura como al psicoanálisis.

En el tercer capítulo, las obras de Cervantes, Kafka y Thomas Mann nos permitirán dar cuenta de una suerte de "crítica del presente" propia de la literatura y su relación con la definición que Zuleta recoge de Georges Lukács de la literatura moderna como “aventura”. A partir de allí se aclarará en qué sentido para el colombiano la experiencia literaria ha de entenderse como "salida" de lo establecido, de lo normado, de la dependencia. Finalmente, se verá aquí cómo la potencia crítica de la literatura y del arte en general establecen una estrecha relación entre las teorías estéticas modernas, tal y como se expresan principalmente en los conceptos de transfiguración y sublimación.

En el cuarto capítulo recogeremos los resultados de la investigación para consolidar y explicar los elementos constitutivos del concepto de experiencia estética que le hemos atribuido a Zuleta. En dirección hacia la conclusión, se contemplarán también las principales consecuencias de su concepción del arte, según la cual la sensibilidad artística es connatural al individuo y a la cultura, lo que implica aspectos problemáticos como la tendencia natural del individuo y de la masa hacia ideologías que se oponen, remplazan o terminan por alienar la naturaleza artística de los unos y de la otra. Al respecto, pondremos a prueba la propuesta central de Zuleta frente al arte, vinculándola al debate que entre Adorno y Jauss se ha dado en torno al principio del goce artístico. Esto lo haremos con el fin de evidenciar cómo la estética del autor colombiano constituye una verdadera oferta de sentido a dicho debate, principalmente porque al conjugar la teoría social de Marx y el principio de realidad o identidad de Freud consigue escapar del presunto subjetivismo y hedonismo que Adorno le atribuye a Kant y a Freud.

En síntesis, dos son los principales propósitos de esta investigación: por un lado, dar cuenta de la manera en que Estanislao Zuleta hizo filosofía desde la literatura y, por otro, como consecuencia principal de un hacer filosófico tan particular, reconstruir y destacar la presencia en la obra de Zuleta de una inconclusa pero sólida teoría estética. En procura de cumplir estos objetivos, tres serán los derroteros: primero, desde el concepto de Lebenswelt y la noción freudiana de sublimación, justificar la compleja relación que tiene lugar entre vida, literatura y filosofía en el pensador colombiano. Segundo, definir el criticismo de Zuleta desde una lectura de Kant, semejante a la de la "ontología del presente" de Foucault, para mostrar cómo desde allí la literatura obtiene un privilegiado lugar en la reflexión filosófica de la modernidad. Y, tercero, desde la novela moderna y la lectura cruzada que hace 
Zuleta de Marx y Freud, ver cómo es posible justificar una noción de experiencia estética según la cual la literatura, como el arte en general, ofrece la posibilidad de reconocer la identidad cultural e individual de forma autónoma y crítica. La construcción de esta noción de experiencia estética atravesará el conjunto de la exposición revelando y articulando paulatinamente el concepto de sublimación de Freud, la estética kantiana, el concepto de transfiguración artística común a Nietzsche y Heidegger y las estrechas relaciones que guardan las nociones de historia y trabajo en Marx con el concepto de elaboración de Freud.

De modo que lo que aquí ofrecemos en consideración pretende aportar a la sistematización de la obra y la filosofía de Zuleta otorgándole al arte y a la literatura un lugar central en su pensamiento, que si bien con frecuencia se ha señalado, pocos trabajos han reparado en los alcances que para la historia de la filosofía tiene una filosofía estética como esta. Lo que querría decir que confiamos en que los aportes del autor colombiano merecen ser reconocidos por la institución filosófica, a pesar de las innegables dificultades que implica lo poco sistematizada que se encuentra su obra.

Si bien esta investigación estuvo destinada a cumplir y concluir con los requisitos de la Maestría en Filosofía de la Universidad del Rosario, nuestro recorrido por la obra y el contexto de Zuleta se remonta a nuestros tempranos años del bachillerato, cuando la lectura al fin cobró para nosotros un verdadero valor para la vida, ya no solo como recurso o práctica académico. Así fue como mucho antes de habernos enfrentado directamente a las magnánimas obras de Dostoievski, Freud o Nietzsche, los textos de Zuleta y su acogedora exposición de estos y otros autores fueron para nosotros una irresistible invitación a estudiar la cultura. Ello parece natural en un medio como el local, pues sumado a que no son muchos los compatriotas que se han destacado en el campo del pensamiento como lo hizo Zuleta, el mito que lo recubre como un autor irreverente y polémico no ha dejado de ser un seductor gancho para jóvenes mentes en formación que, como la nuestra en aquel entonces, se inclinaban más por lo exótico y prohibido que por las recomendaciones del pénsum académico (tal y como lo diagnosticó Zuleta en su mil veces citada reflexión sobre la lectura). Así que con este escrito concluye un largo proceso de formación que es deudor de personas e instituciones a las cuales les queremos agradecer antes de terminar esta presentación introductoria.

En orden de influencia, a quien debemos nuestro primer acercamiento a $\mathrm{Zu}$ leta es a la Escuela Pedagógica Experimental (EPE), institución educativa creada y liderada hace más de 35 años por el reconocido teórico de la educación y pedagogo Dino Segura. La EPE no solo generó las mejores condiciones para comprender lo 
que leímos de Zuleta entonces, sino que, en cuanto proyecto de educación autónoma y crítica, materializa en buena medida las prerrogativas zuletianas sobre la pedagogía. Por otra parte, no podemos dejar de mencionar en estos agradecimientos al recientemente fallecido Jaime Toro Alfonso, porque en nuestro primer año de estudios de filosofía no dudó en presentarnos de nuevo a Zuleta; pero esta vez a la luz de Foucault, Deleuze y el pensamiento francés contemporáneo, del cual Jaime fue siempre un apasionado estudioso e impulsador. También a finales de la década de los noventa, junto a él conocimos a otro de los representantes del pensamiento francés en Colombia, el profesor Adolfo Chaparro Amaya, a quien debemos un especial reconocimiento porque fungió como director y corresponsable de la versión que del presente texto se presentó en 2014 como tesis de maestría. Además de la labor de director de tesis, debemos agradecerle al profesor Chaparro haberse arriesgado a coordinar una investigación sobre un autor que no le era del todo familiar y que no dejaba ni deja de generar escepticismo en los contextos investigativos. A cambio de un amplio estudio de Zuleta, Adolfo Chaparro nos ofreció sus profundos conocimientos en literatura, estética e historia de filosofía, los cuales, junto a su rigurosa y exigente lectura de nuestras distintas versiones, blindaron al escrito de imprecisiones en los numerosos cruces y asociaciones de autores y teorías que constantemente establece.

También en el campo teórico, pero esta vez sí directamente relacionado con la obra y vida de Estanislao Zuleta, debemos agradecer a la Corporación Estanislao Zuleta de Medellín y a la Fundación Estanislao Zuleta de Cali. En cuanto a la primera, no solo nos sentimos en deuda, porque gracias a sus funciones estamos seguros de que este trabajo ha ganado y ganará lectores, pues a la fecha es la organización de mayor reconocimiento y con mayor número de actividades que promueven el legado de Zuleta y su pensamiento crítico. Esto ha sido posible gracias principalmente a Carlos Mario González, discípulo de Zuleta y fundador de dicha corporación, quien tuvo la gentileza de leer y comentar el manuscrito en calidad de jurado de tesis. Respecto a la segunda institución mencionada, nuestros agradecimientos no son pocos, pues gracias a su juiciosa labor, de la mano de Hombre Nuevo Editores, hoy contamos con alrededor de quince publicaciones que recogen buena parte de las conferencias, entrevistas y escritos que dejó el maestro, y gracias a las cuales esta y otras investigaciones sobre el particular han tenido lugar. Vinculado de diversas formas a la sistematización y edición que del pensamiento de Zuleta ha llevado a cabo la fundación de Cali, Alberto Valencia Gutiérrez también ha sido ejemplar para nuestro trabajo, por lo que debemos agradecerle. Tal vez sea él quien de 
manera más rigurosa ha llevado a Zuleta al debate académico, pues con En el principio era la ética... (1996), Estanislao Zuleta o la voluntad de comprender (2005), y sus valiosos artículos, el pensamiento de Estanislao por fin es presentado más allá de su connatural informalidad. A los trabajos de Valencia y su enfoque debemos, pues, reconocer cierta influencia en lo que podríamos llamar "estilo" y, en mayor medida, en cuanto a sus inspiradores señalamientos sobre la relación del arte y el psicoanálisis en el pensador de origen antioqueño. Junto a Valencia y Hombre Nuevo Editores, la difusión del pensador que asumió la Fundación Estanislao Zuleta se debe principalmente al poeta José Zuleta Ortiz, hijo del pensador, a quien debemos agradecer por ello y, además, por su siempre generosa y sincera disposición para atender a quienes, como nosotros, nos interesa conocer de primera mano el entorno íntimo de Estanislao y las consecuencias que tuvo su pensamiento en la vida cotidiana de sus allegados.

Por último, extendemos también nuestros agradecimientos a dos personas que en vida de Zuleta lo acompañaron en sus más valiosas luchas e hicieron parte de sus amigos. Nos referimos al doctor Óscar Espinosa Restrepo y al profesor Hernando Llanos Ortiz (también jurado de la versión de este escrito que sirvió de tesis), quienes desde sus respectivos campos - el primero como reconocido psicoanalista y el segundo desde su incansable difusión de la obra y el pensamiento de Marx - leyeron y comentaron con detenimiento nuestro trabajo, poniendo a nuestro servicio su respetada erudición. Nos queda por reconocer la labor como corrector que en esta y otras ocasiones ha cumplido nuestro buen amigo, colega y compañero de trabajo Camilo Sarmiento Jaramillo, a quien le debemos en parte esta y otras tantas alegrías. 